\title{
Mujeres de Arma: motivaciones para el ingreso al Ejército Nacional de Colombia ${ }^{1}$
}

\author{
Leidy Johana Cabrera Cabrera ${ }^{2}$ \\ Edna Jackeline Latorre Rojas ${ }^{3}$ \\ Escuela Militar de Cadetes "General José María Córdova”
}

\section{Resumen}

La transformación del Ejército Nacional de Colombia por la vinculación de mujeres de Arma desde el 2009 ha motivado diferentes estudios que ponen sobre la mesa el asunto de género como una oportunidad de avance hacia la democratización de la profesión militar. En este artículo, se describen los principales motivos que definieron la decisión de 28 mujeres de Arma para el ingreso al Ejército Nacional de Colombia en dicho año y los retos que ha significado la formación y el trabajo profesional desde entonces hasta la fecha. Los resultados aquí consignados corresponden a un estudio cualitativo que retoma las percepciones de las mujeres de Arma desde su experiencia en la institución militar.

Palabras clave: democratización militar; Ejército Nacional de Colombia; mujeres de Arma; profesión militar

1 Este capítulo presenta de forma conjunta los resultados de dos proyectos de investigación: "Atenea: caracterización sociodemográfica y su relación con la cultura de equidad de los integrantes de la Escuela Militar de Cadetes 'General José María Córdova’. Segunda fase" y "Enfoques diferenciales en el Ejército Nacional: Una propuesta desde las ciencias militares" del Grupo de Investigación en Ciencias Militares, de la Escuela Militar de Cadetes "General José María Córdova" (ESMIC), categorizado en B por Minciencias y registro COL0082556. Los puntos de vista y los resultados de este artículo pertenecen a las autoras y no reflejan necesariamente los de las instituciones participantes.

2 Magíster y especialista en Docencia e Investigación Universitaria (Universidad Sergio Arboleda). Trabajadora social (Universidad de La Salle). Docente e investigadora en ciencias sociales y humanas. Directora del Observatorio Militar de Género de la Escuela Militar de Cadetes "General José María Córdova”. Orcid: https://orcid.org/0000-0002-6398-6933 - Contacto: leidy.cabrera@esmic.edu.co

3 Magíster en Educación e Investigación Universitaria (Universidad Sergio Arboleda). Especialista en Educación e Investigación Universitaria (Universidad Sergio Arboleda). Psicóloga (Universidad Católica de Colombia). Investigadora del Grupo de Investigación en Ciencias Militares, de la Escuela Militar de Cadetes “General José María Córdova”. Orcid: https://orcid.org/0000-0002-0301-9036 - Contacto: edna.latorre@esmic.edu.co 


\section{Introducción}

El proceso de incorporación de mujeres a la carrera militar en diversos países de la región latinoamericana ha permitido

avances en la democratización y modernización de las instituciones de defensa, abriendo la posibilidad de acceder a puestos de comando, y no únicamente a la incorporación de mujeres profesionales (abogadas, médicas, enfermeras, etc.), lo cual sí existía en casi todos los países, al menos desde mediados del siglo XX.

(Donadio, 2010, p. 54)

El Decreto 2129 del 7 de octubre de 1976 permitió el ingreso de mujeres al escalafón militar en la categoría de oficial del cuerpo administrativo. Las primeras doce mujeres que ingresaron a la institución eran especialistas en medicina, arquitectura, derecho, contaduría y administración de empresas, entre otras (Ejército Nacional de Colombia, 2013). Este hecho fue un hito para el Ejército colombiano, porque las mujeres militares pudieron ingresar como profesionales en el ámbito administrativo y con carreras civiles para apoyar las labores del Ejército, aunque sin mando sobre la tropa.

Así pues, el papel de la mujer durante las últimas décadas ha sido crucial en el campo de las labores administrativas de las Fuerzas Militares. Su colaboración ha sido vital en los servicios de apoyo para el combate y el fortalecimiento del cumplimiento de la misión constitucional de las Fuerzas Militares (ONU Mujeres, 2013).

En el 2009, la Escuela Militar de Cadetes "General José María Córdova" (ESMIC) permitió por primera vez el ingreso de 62 mujeres para realizar la carrera de oficial de armas, de forma que ellas tenían la posibilidad de llegar a ocupar cargos como el de general de la república, con lo cual se ponía fin a más de 100 ańos de historia en los que este rol era exclusivamente masculino. En un principio, estas mujeres podían escoger alguna especialidad, como Logística y Comunicaciones, después de tres años de estudio en la ESMIC, y como Arma de combate podían escoger solamente Inteligencia. En el 2001, solo 48 de estas mujeres obtuvieron el título de profesional en Ciencias Militares y el grado de subteniente del Ejército Nacional, a partir de lo cual comenzaron a tener mando sobre un pelotón de soldados y a realizar instrucción militar.

En la actualidad, las mujeres que ingresan a la ESMIC para ser oficiales del Ejército pueden escoger cualquier Arma de combate y realizar todas las actividades que realizan sus compañeros hombres en igualdad de condiciones. La única diferencia es que cuando estas mujeres ascienden al grado de subtenientes y son tras- 
ladadas a cualquier unidad militar de Colombia no se les permite estar en la línea de combate.

Esta situación puede comprobarse en casos particulares. El primero de ellos es el de una teniente de 25 ańos, oficial de comunicaciones del Ejército, que actualmente pertenece al segundo regimiento de las Fuerzas Especiales y es la primera mujer en hacer el curso de tiradora de alta precisión (TAP). También se distingue por ser jefe de Salto y tener curso de combate avanzado (Méndez, 2020). El segundo caso es el de la primera mujer del Arma que se graduó en 2019 de Infantería, una especialidad que durante décadas fue solo para hombres. Esta mujer, de 21 años, está capacitada para desempeñarse como comandante de pelotón en cualquiera de los 46 Batallones de Infantería (Ejército Nacional de Colombia, 2018). Sin embargo, ninguna de las dos, a pesar de haber ganado terreno en lo militar, ha estado en línea de combate.

No obstante, con la vinculación de las primeras mujeres de Arma, es claro que existe un avance importante en la transformación del Ejército Nacional, pues actualmente aquellas que lo decidan pueden desempeñarse en alguna de las Armas de combate (Infantería, Caballería y Artillería, armas que entran en los teatros de operaciones en caso de guerra). A esto se suma el esfuerzo institucional representado por las investigaciones multidisciplinares que buscan comprender, entre otros, las dinámicas, los perfiles y los escenarios que emergen como permanentes desafíos en el campo militar (Perilla-Toro \& Cabrera, 2018).

\section{Profesión o vocación militar}

Para Huntington (1957/1995), los oficiales son profesionales, como los abogados o los médicos. Su singularidad profesional se establece por tres características: la maestría, esto es, los conocimientos expertos y habilidades prácticas especializadas requeridas por el ejercicio del monopolio legítimo en la administración de la violencia; la responsabilidad en el cumplimiento del servicio, que se orienta por ideas y valores apoyados por la sociedad en la que se inscriben; y, por último, el corporativismo propio de la burocracia castrense, constituido en torno a rangos (jerarquías) y cargos (funciones). En cuanto a la dimensión vocacional, esta es también un componente del profesionalismo, tanto de los oficiales como de los suboficiales (Soprano, 2013, p. 74).

En este sentido, la vocación militar se constituye como un factor importante para el éxito de las operaciones militares, debido a que implica la motivación del 
personal de oficiales. La vocación es el impulso para el cumplimiento de objetivos y desarrollo de estrategias. Este impulso para el militar está determinado por el panorama político del país, el bienestar de la población, el direccionamiento estratégico y el soporte jurídico, es decir, en las variables que influyen en la vocación militar y la actitud combativa (Rodríguez, 2013), independientemente del género.

Además, la carrera militar implica un sometimiento, es decir, cierta pérdida de autonomía. Según Julia Evetts (2003), los militares, como individuos y como grupo profesional, solo tienen un control limitado o relativo sobre su proyecto personal y profesional, así como sobre sus trayectorias laborales o planes de carrera. En una sociedad democrática, donde dirigentes políticos y funcionarios civiles conducen la defensa, los militares participan, pero no controlan, en última instancia, la definición de componentes estratégicos de su actividad profesional (educación, operaciones, medios, equipamiento, ascensos de oficiales superiores, etc.). Y si bien, las profesiones civiles como la medicina y la abogacía también están sometidas a formas y grados de regulación estatal, estas gozan de mayor autonomía corporativa.

No obstante, en el caso particular de las mujeres hay un reto adicional frente a su vocación militar: tener que adentrarse en un contexto patriarcal fuertemente apoyado por la misma dinámica social y cultural (De la Hoz, 2017), que da lugar a una situación de patriarcado dentro de las líneas militares y se materializa en forma de una autoridad que establece la división del trabajo dentro de la actividad militar según el género de la persona (Oliva, 2005).

Consecuentemente, comprender cómo una mujer deja su rol socialmente estereotipado para comenzar una carrera militar es una tarea que implica la explicación de nuevas tendencias de lo sociocultural y que se orienta a desmitificar los estereotipos androcéntricos y duales de lo femenino y lo masculino.

\section{La teoría ecológica de Bronfenbrenner en los proyectos de vida}

Bronfenbrenner (1987) plantea que, para alcanzar la comprensión sobre el desarrollo y cambio de conducta de los seres humanos, es necesario reconocer su interacción con el entorno próximo y profundizar en el significado que le atribuyen desde las acciones y el discurso a sus vivencias y experiencias. Este autor asegura que "los ambientes naturales son la principal fuente de influencia sobre la conducta humana" (p. 37), de esta manera ofrece un entendimiento, como él mismo lo denomina, "ecológico" de las contingencias que pudieran demarcar los 
sistemas humanos al interpretarlos como una sucesión de estructuras seriadas (Parra \& Rubio, 2017).

Desde su propuesta, Bronfenbrenner (1987) ubica seis niveles en los que se organiza este tipo de relaciones, de los cuales se destacan cuatro:

- Microsistema. En este nivel se incluyen los roles, relaciones y comportamientos en los contextos cotidianos, como el hogar, el trabajo, los amigos y cualquier otro espacio en el que el individuo se vea inmerso de manera frecuente o cotidiana (Parra \& Rubio, 2017; Torrico et al., 2002).

- Mesosistema. Aborda los entornos en los que el sujeto participa de manera activa y regular, los círculos sociales a los cuales pertenece, los cuales están a su vez integrados por otros microsistemas (Torrico et al., 2002). En este proceso se incluyen los contextos en los cuales el individuo se ve inmerso de manera involuntaria, que implican relaciones indirectas o circunstanciales (Parra \& Rubio, 2017).

- Exosistema. Se refiere a los espacios donde el sujeto no influye de manera activa, aunque se ve afectado de manera directa por lo que ocurre (Parra \& Rubio, 2017; Torrico et al., 2002).

- Macrosistema. Es el nivel mayor, que incluye los ámbitos sociales, culturales, políticos y económicos en los que interactúan los sujetos. La comprensión de este nivel permite reconocer las percepciones e imaginarios sociales más complejos que enfrenta el sujeto (Bronfenbrenner, 1987; Parra \& Rubio, 2017).

Estos niveles configuran una forma de comprender a los sujetos en relación con los diferentes factores que intervienen en sus interacciones y ambientes. Para este estudio se retoman estos cuatro ejes (micro-, meso-, exo- y macrosistemas) que, al ser llevados al escenario militar, se convierten en una aproximación interesante para analizar las percepciones de las oficiales militares frente a los diferentes factores que han influido en su incorporación al Ejército.

Es importante considerar que cada sujeto construye su realidad a través de la interacción con el contexto (ambiente), por lo que el lenguaje y el discurso son herramientas culturales claves para que dicha interacción se produzca (Colás $\&$ Jiménez, 2004). En este sentido, el uso de un lenguaje con características fácilmente reconocibles, bien sea en su manifestación oral o escrita, es una de las principales señas de identidad de los ejércitos (Álvarez, 2018). Álvarez también indica que la génesis y desarrollo de este lenguaje obedece a motivaciones que buscan mejorar la eficacia mediante una comunicación que, además de ser fiable, contribuya al 
mantenimiento de la disciplina y sirva para mostrar algunas de las cualidades personales que distinguen a los buenos soldados. De ahí la importancia de abordar el lenguaje dentro del análisis de las motivaciones de las mujeres de Arma para ingresar al Ejército Nacional de Colombia como un componente propio de la(s) identidad(es) militar(es).

\section{El lenguaje en la construcción de identidades militares: el lugar del género}

El lenguaje no es un fenómeno individual, es un fenómeno social. Un solo individuo, por sí mismo, no puede producir lenguaje. El lenguaje surge en el proceso de interacción social, en el juego colectivo de individuos que coordinan acciones juntos (Echeverría, 2003, p. 344). A través de la comunicación, en el lenguaje y en el discurso, se afirma, se niega y se cuestiona con el fin de entrecruzar la pluralidad de acciones e intereses mediante la negociación, la persuasión y el compromiso; de esta manera, se materializa el ejercicio del consenso.

En palabras de Echeverría (2003), el lenguaje es el consenso de un conjunto de distinciones para coordinar acciones conjuntas sobre una base estable, es lo que constituye una comunidad. A su vez, una comunidad está organizada como un sistema de coordinación de acciones entre sus miembros, con base en un lenguaje compartido. Cuando no hay juegos integradores, cuando los individuos dejan de coordinar acciones entre ellos, la comunidad, por definición, se desintegra. De acuerdo con el mismo autor, la persona construye, desde el lenguaje, una serie de dominios que le permiten desempeñarse en diferentes escenarios, como puede evidenciarse en la tabla 1.

Tabla 1. Dominios de la persona

\begin{tabular}{ll}
\hline \multicolumn{1}{c}{ Tipo de dominio } & \multicolumn{1}{c}{ Descripción } \\
\hline $\begin{array}{l}\text { La persona como dominio } \\
\text { experiencial }\end{array}$ & $\begin{array}{l}\text { Incluye las experiencias como componentes básicos de la } \\
\text { vida; cualquier cosa que se hace, se hace desde las expe- } \\
\text { riencias. }\end{array}$ \\
$\begin{array}{l}\text { La persona como dominio } \\
\text { discursivo }\end{array}$ & $\begin{array}{l}\text { Aborda la persona como una narrativa o historia que se } \\
\text { cuenta sobre quienes somos. Tales historias siempre se } \\
\text { sustentan en algunos juicios fundamentales que se han } \\
\text { llamado juicios maestros. Estos juicios suelen versar sobre la } \\
\text { persona, los otros, el mundo y el futuro. }\end{array}$ \\
\hline
\end{tabular}




\section{Tipo de dominio}

La persona como dominio performativo

La persona como dominio moral

La persona como dominio emocional

\section{Descripción}

La forma en que se actúa no siempre puede ser inferida de una historia o un grupo de historias que la genere. Se realizan ciertas cosas solo porque esa es la forma en que hemos visto a la gente hacerlas, pero generalmente ni siquiera sabemos que la forma en que hacemos las cosas, la forma en que enfrentamos la vida, es solo una forma posible de hacerlo, sino que la tomamos como la forma obvia y natural de hacerlas; las damos por sentadas (p. 351).

Desde este dominio, se concibe la persona como un conjunto de declaraciones acerca de las acciones que pueden, no pueden y deben ser realizadas en determinadas circunstancias. Estas declaraciones pueden remontarse a las narrativas y a las prácticas, pero aun así pueden tener algún grado de autonomía. Individuos que provienen de los mismos discursos históricos y que comparten las mismas prácticas sociales pueden tener límites morales diferentes (p. 353).

Las emociones pueden reconstruirse lingüísticamente y también pueden cambiar debido a interacciones lingüísticas. Son consideradas, sin embargo, un dominio distinto del lenguaje. El lenguaje puede afectar las emociones, así como las emociones pueden afectar el lenguaje. Debido al estado emocional, se entablan ciertas conversaciones y no hay disposición para otras (p. 357).

Fuente: Elaboración propia con base en Echeverría (2003)

Los efectos del lenguaje son reconocibles en la construcción del género como variable de la identidad social del individuo (Gregorio, 2000, citado por Butler, 2007). En este sentido, la identidad y las identidades femeninas se construyen y deconstruyen en el ejercicio del lenguaje en los diferentes niveles de interacción y tipo de dominio.

Por tal motivo, el lenguaje comprende un campo interesante para describir procesos orientados hacia la identificación de inequidad, desigualdad, estereotipos y violencias de género. Así, los estudios de lenguaje y su relación con lo femenino son bastantes, generalmente orientados, por una parte, hacia la discusión del sexismo lingüístico a nivel léxico, morfológico y sintáctico, y, por otra parte, al lenguaje no sexista (González \& Delgado de Smith, 2013).

La forma en que desde el lenguaje se aborda la identidad femenina tiene que ver con poder decidir qué historias contar y transmitir. Esto se vuelve fundamental para una presencia igualitaria de lo femenino en las representaciones y percepciones 
políticas, sociales y culturales (Marín, 2015) desarrolladas en un contexto como el militar y presentes en sus dinámicas sociales.

\section{Método}

Este capítulo describe las principales motivaciones para la vinculación al Ejército de 28 de las oficiales de Arma incorporadas en la primera cohorte (2009), tomando sus narrativas como eje central de análisis. Observar, conversar y escuchar sus historias permitió dar sentido al mundo que construyen y resignificarlo, para comprender lo que hay detrás de los relatos en relación con mitos, costumbres, tradiciones, prejuicios y verdades (Uribe, 1993).

El estudio se realizó mediante un enfoque cualitativo-descriptivo, en el que se retomaron técnicas de revisión documental y se revisó un grupo focal. El grupo focal buscó identificar los hitos, en la historia de vida de mujeres oficiales, que determinaron su vinculación al Ejército. La duración fue de 120 minutos en las instalaciones de la Escuela de Armas Combinadas. Las participantes del grupo focal fueron 28 mujeres, vinculadas desde el año 2009 al Ejército, específicamente, en la Escuela de Armas Combinadas para realizar su curso de ascenso de teniente a capitán. De las 28 oficiales que participaron del proceso, catorce oficiales son solteras, ocho son casadas, tres son separadas y tres viven en unión libre. La mayoría de las oficiales no tienen hijos (16), las demás manifestaron tener hijos. El rango de edad de las entrevistadas está entre los 28 y 31 años. Un poco más de la mitad se encontraron en el rango de 27 y 28 años (53\%). Los instrumentos y el proyecto en general tuvieron aprobación por parte del Comité de Ética de la Escuela Militar de Cadetes "General José María Córdova".

\section{Resultados y discusión}

\section{El ingreso a la profesión militar}

El ingreso a la carrera militar generalmente tiene que ver con factores intrínsecos o extrínsecos que influyen en la toma de decisiones y enmarcan el proyecto de vida. Para el caso de las mujeres en las Fuerzas Militares, este proceso tuvo también relación con las "necesidades de reclutamiento de personal, en un contexto en el cual las profesiones civiles ganaron adhesión no sólo por la oferta económica sino también por la pérdida de nitidez que antes tenían las funciones y misiones de la carrera militar" (Donadio, 2010, p. 55). Desde los relatos generados, las principales 
motivaciones para la vinculación al Ejército de estas mujeres estuvieron determinadas por cuatro niveles según la teoría ecológica de Bronfenbrenner (1987) (tabla 2).

Tabla 2. Teoría ecológica: motivaciones para ingresar al Ejército Nacional

\begin{tabular}{ll}
\hline Microsistema & $\begin{array}{l}\text { Gusto personal } \\
\text { Admiración por la profesión } \\
\text { Deporte como proyecto de vida }\end{array}$ \\
Mesosistema & Tradición familiar \\
Exosistema & Búsqueda de un trabajo estable \\
Macrosistema & Conflicto armado colombiano \\
\hline
\end{tabular}

Fuente: Elaboración propia con base en Bronfenbrenner (1987)

En el caso de las oficiales, sus motivaciones para la vinculación abarcan intereses personales, tradición familiar, búsqueda de trabajo estable, admiración por la profesión y situación familiar como víctima de conflicto armado, todos ellos ubicados en su mayoría en el nivel microsistémico.

Sobre la motivación por la vinculación, cinco consideraron como primera opción realizar la carrera militar, las demás tenían como primera opción avanzar en estudios universitarios de medicina, biología, derecho, relaciones internacionales y administración de empresas.

Casullo et al. (2006) señala que las elecciones y recuerdos de cada persona se ajustan a su esquema cognitivo, de forma que eligen aquello que es consistente con su autoimagen, de manera que lo que perciban los demás esté en concordancia con el propio autoconcepto. Dado el efecto de la familia sobre el autoconcepto, se muestra una clara influencia de esta sobre la decisión de vincularse al Ejército. Como aspecto que permite la comprensión de las influencias que pudieron recibir las oficiales, se indagó por la persona o figura pública que admiraba antes de ingresar al Ejército: las respuestas muestran que en su mayoría esta persona fue una mujer de ficción, una artista o una política (figura 1).

En su narrativa, las oficiales mencionan la necesidad de configurar referentes femeninos en el contexto militar lo que actualmente no se presenta. Este hecho resulta llamativo pues corresponde a la tardía incorporación formal de las mujeres a las armas. Es posible que, en instituciones donde el ejemplo personal y la identificación con personas caracterizadas por sus hazañas históricas jueguen un papel importante (Lupano et al., 2008, p. 212). 


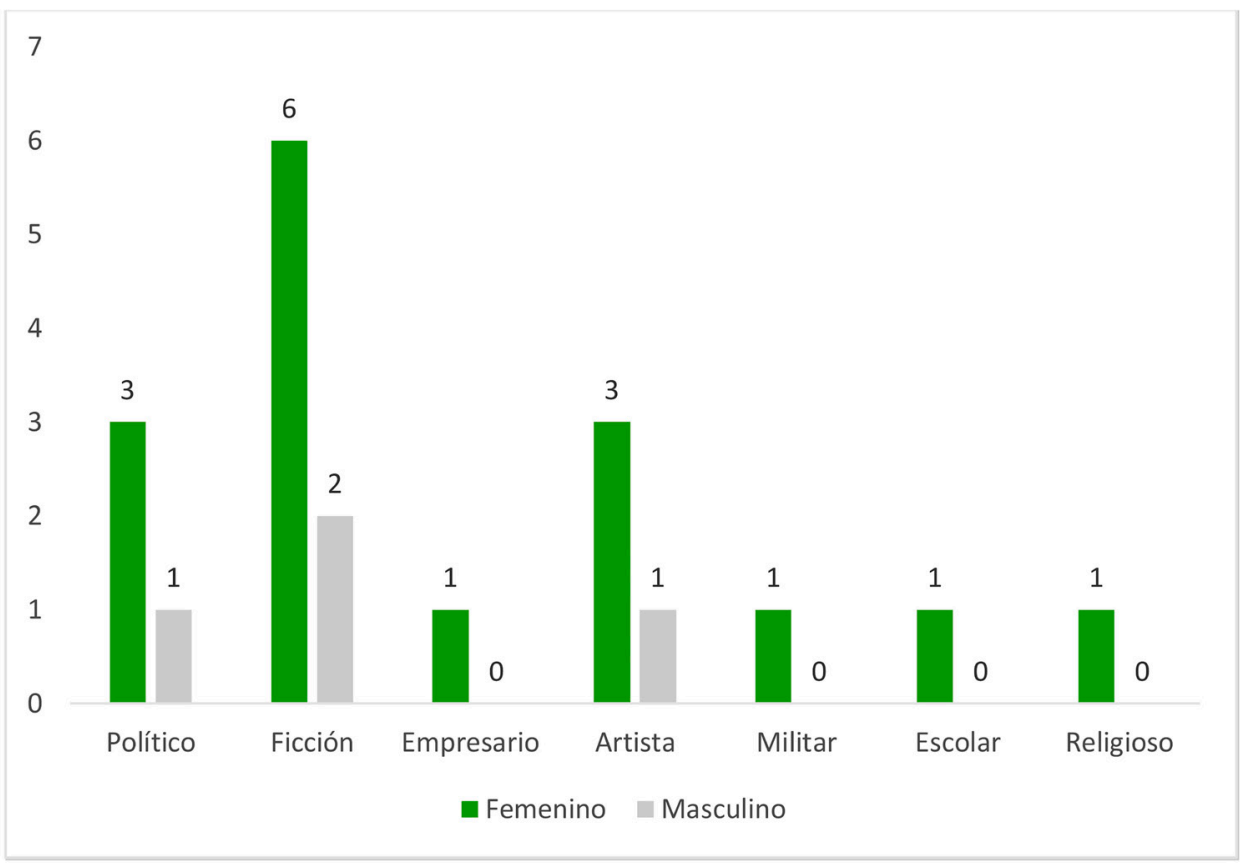

Figura 1. Persona o figura pública admirada por las mujeres entrevistadas.

\section{Concepciones sobre "ser militar" desde el lenguaje}

El oficial formado en la ESMIC es competente como líder, administrador, instructor y comandante de pelotón. Está dotado de habilidades comunicativas, técnicas y tácticas adecuadas para el planeamiento, conducción y ejecución de maniobras terrestres en un ámbito multimisión. Ostenta una sólida fundamentación en valores, principios y virtudes militares, así como un elevado compromiso ciudadano. Está capacitado para el análisis de situaciones complejas, toma de decisiones y solución de conflictos. Tiene actitud innovadora, apoyada en la ciencia y la tecnología; así mismo, es poseedor de una óptima condición física, que le permite resistir las exigencias propias del servicio (Escuela Militar de Cadetes "General José María Córdova”, 2019).

La misión como mujeres militares no está dada de forma específica para ellas. Existe un solo perfil de oficial del Ejército Nacional, sin distinción de género. Sin embargo, en el quehacer militar se han destacado escenarios en los que ellas resaltan su ejercicio profesional con un sello específico que va más allá del apoyo a las operaciones desde las diferentes armas. En alguno de los relatos, una de las participantes menciona: "Lo que quiere el Ejército es hacerse querer más por la población civil, 
creo que las mujeres tenemos mayor capacidad y credibilidad para lograr esto [...]. Somos más amenas para la población civil y podemos entrarle más fácil a un campesino". Lo que se trasmite desde estas perspectivas ubica a las mujeres militares en el macrosistema de la ruralidad colombiana. Estos sellos, si son vistos de forma simplista, pueden configurar estereotipos que las ubican en un único perfil militar; pero si son vistos de una manera integral, enriquecen el cumplimiento de la misión constitucional del Ejército.

En general, la experiencia de las 28 oficiales en el Ejército ha tenido componentes positivos y negativos. Se destaca, por ejemplo, la productividad en términos profesionales, la experiencia y el hecho de romper con paradigmas que han sido fundamentalmente impuestos para hombres. Así, la escasez de ejemplos femeninos de liderazgo efectivo puede ocasionar algunas incertidumbres en la incorporación y formación de las cadetes mujeres, con lo cual se perpetúa la identificación de modelos masculinos de liderazgo (Lupano et al., 2008, p. 212).

Las oficiales coinciden en que no desean privilegios ni contemplaciones, sino más bien igualdad de oportunidades. "Hombres y mujeres son parte de un cuerpo profesional del Estado, en el que resta todavía trabajar sobre criterios de promoción y ascenso por capacidades independientemente del género" (Donadio, 2010, p. 55).

Mantenerse en el Ejército se ha convertido en una decisión compleja, sobre todo, cuando de la conformación familiar se trata, pues, contrario a otras profesiones, manifiestan que las dificultades para llevar una familia son cada vez mayores, especialmente por la demanda del tiempo y energía que requiere la profesión: "Esto es incompatible con la vida familiar..., con los hijos..." (MM3). Otra replica: "... pero esto ya lo sabíamos al ser oficial de Arma, usted sabe a lo que se metió” (MM4).

Los hallazgos muestran que sus narrativas en cuanto a su rol de mujeres militares se centran en un dominio de lenguaje experiencial y emocional. Desde su discurso, se evidencia que, al momento de su incorporación, fue claro que no existió la planeación necesaria, por lo que vivieron en una constante improvisación. Resulta interesante observar que cuando se habla de incorporación femenina, a pesar de contar con las condiciones físicas de infraestructura en el alojamiento para el desarrollo y esparcimiento de hombres y mujeres, y a pesar de contar con igualdad en la formación académica, no hay una proyección clara en la vida profesional de ellas cuando se habla con las futuras capitanas. Frases como "Somos lo que se coloca cuando nadie más esta para poner", "Nos tienen tapando huecos, mientras ellos salen a área" o "Llevo tres años dando instrucción a los soldados" (declaraciones del grupo de tenientes en servicio activo pertenecientes al grupo focal) muestran una necesidad clara de un plan de carrera para ellas. 
Se evidencia, entonces, que la construcción social del proyecto de vida está arraigado a una misión institucional que, muchas veces, comienza con ideas altruistas, como enfrentar al enemigo, dar la vida por el país y desear estar en el área para dar lo mejor de sí. Además, las experiencias como militares implican, desde un comienzo, el desarrollo de estereotipos de hombría en escenarios altamente violentos que se asemejan a los ideales fantásticos propios de la nińez, como ser superhéroes y vencer a los criminales. Otro aspecto importante en el lenguaje de construcción militar es el deseo de tener una carrera permanente que haga más fácil tener un empleo y no entrar a competir en la baja demanda laboral que tiene el país, para garantizarse una estabilidad económica.

Forero Ángel (2017) plantea que las narrativas de los militares explican que la motivación del ingreso a la vida militar subyace principalmente en la vocación, asociada a la lucha contra el enemigo, a la formación para pasar del cuerpo civil al militar, al entrenamiento continuo, a la tecnificación en el uso de armas y a los horarios.

Es evidente que la formación desde la ESMIC, la especialidad del Arma y los cursos de ascenso son iguales, pero las oportunidades de carrera no lo son. Ellas inician queriendo ser guerreras, empoderadas y con la ilusión de servir a la patria como un soldado más. Así mismo, existe la necesidad de abrir camino para las mujeres que vienen atrás.

\section{Una agenda de género}

En la actualidad existe un creciente interés científico por los estudios de género en las Fuerzas Armadas (Lupano et al., 2008; Cronin, 2003; Holm, 1992; Firestone \& Stewart, 2001; Shields, 1988). En este sentido, la incorporación de una perspectiva de género en la formación militar requiere tomar en cuenta los mecanismos de marginación y exclusión (Badaró, 2010), como las posibilidades de agencia para las mujeres en el ámbito militar. Así, como parte de la construcción de una agenda de género, se constituyó el primer Observatorio de Género, Seguridad y Fuerza Pública en la ESMIC.

\section{El Observatorio de Género, Seguridad y Fuerza Pública de la ESMIC}

El Observatorio de Género se constituyó a partir de la Resolución 2744 de 2013, con el nombre de Observatorio de Equidad de Género, Seguridad y Fuerza Pública. Su definición incluyó consideraciones específicas, dentro de las que se encuentra la incorporación del primer curso de aspirantes femeninas para formarse como oficiales de las armas. De esta manera, surgió el interés en medir el impacto de la presencia del personal femenino y su rol en actividades militares. Esto le ha permitido al Ejército Nacional y a la ESMIC fijar políticas y procesos que regulan 
el papel que debe tener la mujer dentro de la Fuerza, así como su desempeño, funciones y actividades en situaciones de conflicto, posconflicto e intervención en operaciones humanitarias encaminadas al mantenimiento de la paz y al orden social. Todo ello, en su condición de oficial de la república y en concordancia con el perfil genérico del egresado de la ESMIC.

Es importante aclarar que el Observatorio de Género, Seguridad y Fuerza Pública, dentro de sus políticas, concibe la mujer como una protagonista proactiva de cambios sociales y como líder activa de la Fuerza Pública. Su posición se corresponde con la política pública de equidad y género (Conpes, 2015) y con las resoluciones del Consejo de Seguridad de la ONU (2020) que obliga a las instituciones públicas de las naciones a abrir discusiones sobre asuntos de género.

Durante el 2018, el Observatorio de Género de la ESMIC trabajó de acuerdo con dos vertientes dentro de su plan de desarrollo: por un lado, el Programa E-Atenea como estrategia de intervención en la cultura de equidad dentro de la comunidad académica, por otro, el desarrollo de proyectos de investigación formal que permiten la transversalización del enfoque de género en los procesos de formación.

Según los resultados de estas dos vertientes, las mujeres deberían integrarse en los espacios masculinos para lograr una medición de sus alcances e indicadores de integración en la institución militar. Si bien las mujeres suelen encontrar mayores obstáculos que los hombres en espacios predominantemente masculinos como las Fuerzas Armadas, sería un error deducir que lo que hay que hacer es buscar la forma de que las mujeres se integren sin revisar los mecanismos institucionales para ello (Badaró, 2010).

Por último, Badaró también considera que los mecanismos formales e informales que obstaculizan la "integración" de las mujeres pueden producir, además, la desintegración entre los supuestamente integrados (los hombres) o, por el contrario, habilitar nuevas formas de integración para todas las mujeres y todos los hombres de la institución. Es decir, estos mecanismos pueden habilitar nuevas formas de agencia que a su vez introduzcan posibilidades de cambio institucional.

\section{Reflexiones finales}

El Ejército Nacional, como institución de formación, avanza hacia la comprensión de las nuevas dinámicas que caracterizan la vida militar de las mujeres de Arma. Sin embargo, ellas continúan expresando la necesidad de tener mayor participación en la toma de decisiones que afectan directamente su rol profesional, no con el ánimo de adquirir beneficios, sino con la intención de configurar desde 
sus mejores capacidades el fortalecimiento de la misión institucional. Este proceso supone analizar el perfil de quienes forman parte de las instituciones militares para ampliar sus oportunidades de servicio con roles claramente definidos.

De acuerdo con los resultados de la encuesta aplicada para esta investigación, las principales motivaciones de las mujeres de Arma para ingresar al Ejército incluyen aspectos relacionales de su microsistema, es decir, su familia, escuela o grupos de amigos que marcaron de forma determinante la decisión de proyecto de vida como oficiales. La incorporación de mujeres puede transformarse en una oportunidad para problematizar otras dimensiones de la institución y para implementar medidas y políticas orientadas a atender cuestiones de género que produzcan efectos que trasciendan este campo (Badaró, 2010).

Así mismo, la persona, como dominio experiencial planteado por Echeverría (2000), refleja el componente principal del discurso expresado por las oficiales, pues las experiencias individuales son su principal forma de comunicarse.

De acuerdo con los resultados de esta investigación, es claro que el estudio sobre temas de género en el ámbito militar corresponde a un componente de la identidad militar misma, enmarcado en las transformaciones actuales que vive el Ejército en su interior y su relación con la sociedad y el Estado. Las formas de relación que se reflejan en las percepciones de las oficiales pasan por debates morales y por la construcción de significados de lo que implica estar en el mundo militar y fuera de él.

La mirada al interior de las instituciones militares es necesaria como proceso investigativo, toda vez que el ejercicio retrospectivo genera un constante avance hacia nuevas posibilidades de consolidación, para lo que se requiere de esfuerzos importantes en los que se pongan en evidencia problemas y falencias internas.

\section{Referencias}

Álvarez, J. (2018). De re diplomatica militari: archivos y documentos de la defensa. Universidad Complutense de Madrid. https://www.ucm.es/documad/de-re-diplomatica-militari-archivos-y-documentos-de-la-defensa

Badaró, M. (2010). Mujeres militares y políticas de género en las Fuerzas Armadas: algunas reflexiones. En Género y Fuerzas Armadas. Algunos debates teóricos y prácticos, Género y Fuerzas Armadas. Ministerio de Defensa y Fundación Friedrich Ebert.

Bronfenbrenner, U. (1987). La ecología del desarrollo humano. Paidós.

Butler, J. (2007). El género en disputa el feminismo y la subversión de la identidad. Ediciones Paidós.

Casullo, M., Cayssials, A., Fernández, M., Arce, J., \& Álvarez, P. (2006). Proyecto de vida y decisión vocacional. Paidós. 
Colás, P., \& Jiménez, R. (2004). La cosmovisión cultural de género del profesorado de Secundaria. Cultura y Educación, 16(4), 419-433.

Conpes. (2015). Politica pública nacional de discapacidad e inclusión social [Documento Conpes Social, 166 ]. https://colaboracion.dnp.gov.co/CDT/Conpes/Social/166.pdf

Consejo de Seguridad de la ONU. (2020). Paz y seguridad. https://www.un.org/securitycouncil/es

Cronin, S. (2003). Military psychology: An introduction. Pearson.

De la Hoz Flórez, M. P. (2017). La mujer en las Fuerzas Armadas de Colombia y España: agente de reconstrucción social durante el posconflicto [Tesis de pregrado, Universidad Católica de Colombia]. Repositorio Institucional. https://repository.ucatolica.edu.co/handle/10983/15790

Donadio, M. (2010). La cuestión de género y la profesión militar. En N. Garré (Comp.), Género y fuerzas armadas. Algunos debates teóricos y prácticos. Ministerio de Defensa; Fundación Friedrich Eberd.

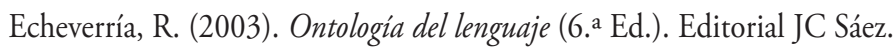

Ejército Nacional de Colombia (2013). La fuerza también tiene heroínas: el papel de la mujer en el Ejército colombiano. https://www.ejercito.mil.co/?idcategoria=349399

Ejército Nacional de Colombia. (2018). La primera mujer de Infantería en la historia de Ejército Nacional. https:/www.esmic.edu.co/sala_prensa/noticias/la_primera_mujer_infanteria_2681_2681

Escuela Militar de Cadetes “General José María Córdova”. (2019). Perfil profesional. ESMIC.edu.co. https://www.esmic.edu.co/index.php?idcategoria $=466$

Evetts, J. (2003). Explaining the construction of professionalism in the military: History, concepts and theories. Revue Française de Sociologie, 44(4), 759-776. https://doi.org/10.2307/3323236

Firestone, J. M., \& Stewart, J. B. (2001). Trends in gender and racial equity in retention and promotion of officers. En M. Dansby, J. Stewart, \& S. Webb (Eds.), Managing diversity in the military (pp. 231-256). Transaction.

Forero Ángel, A. M. (2017). El Ejército Nacional de Colombia y sus heridas: una aproximación a las narrativas militares de dolor y desilusión. Antipoda. Revista de Antropología y Arqueología, 29, 41-61. https://dx.doi.org/10.7440/antipoda29.2017.02

González, M. C., \& Delgado de Smith, Y. (2016). Lenguaje no sexista: una apuesta por la visibilización de las mujeres. Comunidady Salud, 14(2), 86-95.

Holm, J. (1992). Women in the military: An unfinished revolution. Presidio.

Huntington, S. (1957/1995]. El soldado y el Estado. Teoría y práctica de las relaciones civico-militares. Grupo Editor Latinoamericano.

Lupano, M. L., Castro, A., \& Martina, M. (2008). Prototipos de liderazgo masculino y femenino en población militar. Revista de Psicología (Lima), 26(2), 195-218.

Marín, S. (2015). Mujer, escrituras y comunicación [Tesis doctoral, Universidad de Sevilla]. https:// idus.us.es/bitstream/handle/11441/38434/APROXIMACIONES\%20DESDE\%20UN\%20 MARCO\%20TE\%D3RICO\%20(FINAL).pdf? sequence=1 \&isAllowed=y

Méndez, A. (2020). Mamá de 2 niñas, paracaidista y la primera francotiradora del Ejército. El Tiempo. https://www.eltiempo.com/justicia/servicios/la-historia-de-la-primera-mujer-francotiradora-del-ejercito-469474

Oliva, A. (2005). Debates sobre el género. En C. Amorós, \& A. de Miguel (Ed.), Teoría feminista de la ilustración a la globalización (pp. 15-60). Minerva. 
ONU Mujeres. (2013). Plan estratégico de la Entidad de las Naciones Unidas para la Igualdad de Género y el Empoderamiento de las Mujeres 2014-2017. https://colombia.unwomen.org/es/como-trabajamos/estrategia-pais-2017-2019

Parra, P., \& Rubio, Y. (2017). Una mirada desde el modelo ecológico de Bronfenbrenner de dos historias de sujetos que se convirtieron en padres/madres durante su adolescencia [Tesis de pregrado]. Fundación Universitaria Los Libertadores.

Perilla-Toro, L. E., \& Cabrera, L. J. (2018). Caracterización del liderazgo militar desde una perspectiva de género. En A. Fernández-Osorio, \& J. Latorre (Eds.), La construcción del rol de la mujer militar (pp. 67-91).Escuela Militar de Cadetes "General José María Córdova”.

Rodríguez, E. (2013). Escenarios y tendencias de la estructura de la Fuerza Aérea Colombiana con relación al panorama político del pais [Trabajo de grado de especialización]. Universidad Nueva Granada. Repositorio Digital. https://repository.unimilitar.edu.co/handle/10654/13652

Shields, P. M. (1988). Sex roles in the military. En C. C. Moskos, \& F. R. Wood (Eds.), The military: More than just a job? (pp. 99-113). Pergamon-Bassey.

Soprano, G. (2013). Ser militar en la argentina del siglo XXI: entre una vocación, una profesión y una ocupación. Avá. Revista de Antropología, 23, 71-95.

Torrico, E., Santin, C., Monserrat, A., Villas, A., Menéndez, S., \& López, J. (2002). El modelo ecológico de Bronfenbrenner como marco teórico de la psicología. Anales de Psicología, 18(1), 45-59. http://www.um.es/analesps/v18/v18_1/03-18_1.pdf

Uribe, M. T. (1993). Investigación cualitativa. Instituto de Estudios Regionales (INER) de la Universidad de Antioquia. 